\title{
Ensayo clínico multicéntrico para la validación de la aplicación Glikal en la ayuda al manejo de la hiperglucemia en comparación con la práctica clínica habitual.
}

\author{
César Carballo Cardona ${ }^{1 *}$, Paloma Gallego Rodríguez ${ }^{2}$, Carmen María Olmeda Brull ${ }^{3}$, Ricardo A. Juárez González ${ }^{4}$, \\ Jesús María Díaz del Campo Córdoba ${ }^{5}$, Roberto del Río lbáñez ${ }^{6}$, Rafael Cuervo Pinto ${ }^{7}$, Luis Pérez Ordoño ${ }^{8}$, Fernando \\ Camón Iglesias ${ }^{9}$, Vanessa Heras Fernández ${ }^{10}$, Esther Álvarez Rodríguez ${ }^{11}$, Teresa Agudo Villa ${ }^{11}$, Juan Vila Santos ${ }^{8}$, Luz \\ Tamara Vázquez Rodríguez ${ }^{8}$, Isabel Gallego Rodríguez ${ }^{1}$, Cristina Fernández-Pérez ${ }^{12}$.
}

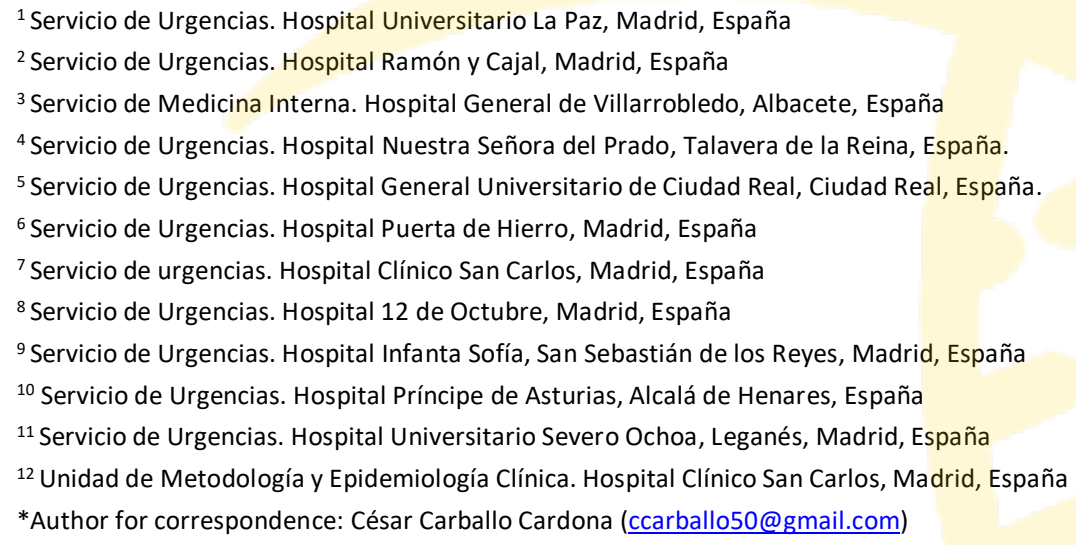

\section{ABSTRACT}

Ensayo clínico multicéntrico, para la validación externa de una aplicación informática (GLIKAL) demostrando su superioridad frente a la práctica clínica habitual en el control de la hiperglucemia en urgencias. Los pacientes que no sean vistos por el equipo investigador, conformarían el grupo tratado por práctica clínica habitual (PCH) y los pacientes incluidos por el equipo investigador conformarían la cohorte de pacientes tratados por GLIKAL. Se recogieron 3 determinaciones de glucemia al día durante 48 horas tras el ingreso, además de los episodios de hipoglucemia y los días de ingreso de ambos grupos. Se reclutaron en total 183 pacientes, de entre 18 a 95 años de los cuales 86 fueron tratados con GLIKAL y 97 según la PCH. Las medias de las glucemias en las primeras 24 horas, fue de 178.56 (59.53) mg/dl del grupo GLIKAL y de 212.93 (68.23) mg/dl del grupo PCH $(p<0.001)$. Mientras que en el segundo día, el grupo GLIKAL tuvo una media de glucemia de 173.86 (58.86) mg/dl y el grupo PCH de 196.37 (66.60) $\mathrm{mg} / \mathrm{dl}(\mathrm{p}=0.017$ ). En ambos días, los pacientes que lograban mantener cifras de glucemia por debajo de $180 \mathrm{mg} / \mathrm{dl}, \mathrm{era}$ de más del 60\% para el grupo GLIKAL, mientras que el grupo PCH se mantenía entre el 33 y el $43 \%$, siendo esta diferencia estadísticamente significativa para ambos días. Estos resultados demuestra la superioridad en resultados de disminución de glucemia de la aplicación GLIKAL frente a la práctica clínica habitual.

Palabras claves: Glucemia; Diabetes Mellitus; Hiperglucemia

Recibido 20 de marzo, 2017; Aceptado 24 de mayo, 2017; Publicado 20 de julio, 2017.

Copyright: (c) 2017 Authors. This is an open-access article distributed under the terms of the Creative Commons Attribution License, which permits unrestricted use, distribution, and reproduction in any medium, provided the original author and source are credited.

Editor: Antonio J. Carcas Sansuán

Citar como: Carballo Cardona C., Gallego Rodríguez P., Olmeda Brull CM., Juárez González RA:, Díaz del Campo Córdoba JM., Del Río Ibáñez R., Cuervo Pinto R., Pérez Ordoño L., Camón Iglesias F., Heras Fernández V., Álvarez Rodríguez E., Agudo Villa T., Vila Santos J., Vázquez Rodríguez LT., Gallego Rodríguez I., Fernández-Pérez C. Ensayo clínico multicéntrico para la validación de la aplicación Glikal en la ayuda al manejo de la hiperglucemia en comparación con la práctica clínica habitual. IBJ Plus 2017 1(1):e0005.

Fuentes de financiación: The author(s) declared that no grants were involved in supporting this work.

Conflictos de intereses: Los autores declaran que no existen conflictos de intereses en relación con el presente trabajo. 


\section{Introducción}

La diabetes mellitus (DM) tipo 2 se ha convertido en uno de los problemas sanitarios más graves de nuestro tiempo. Sus proporciones son ya epidémicas en la mayor parte del mundo: se estima que actualmente existen 246 millones de personas afectadas en todo el planeta, una cifra que puede llegar a los 380 millones en el año 2025 si se cumplen las últimas predicciones ${ }^{1-}$ 6 .

A nivel mundial, se estima que los costes sanitarios debidos a la DM ascendieron a 548 mil millones de dólares en $2013^{7}$ (un 11\% del gasto sanitario total en adultos). En España, la diabetes es la principal enfermedad crónica, y sus costes directos pueden suponer entre un $7 \%$ y un $13 \%$ del gasto total del Sistema Nacional de Salud ${ }^{5}$. Una de las líneas de ejecución del plan estratégico de atención a pacientes con enfermedades crónicas, desarrolladas por la Consejería de la Comunidad de $\mathrm{Madrid}^{8}$, se basa en el desarrollo de herramientas informáticas de apoyo a pacientes y profesionales, y en concreto, se hace énfasis en las ayudas a la prescripción mediante herramientas (TIC) de ayuda a la gestión clínica, dirigidas al manejo de la prescripción farmacológica y sus interacciones, así como a la gestión coordinada entre niveles asistenciales de la terapéutica del paciente crónico (conciliación). Estas herramientas, han demostrado ser rentables (útiles y costo-efectivas) ${ }^{9}$ ${ }^{10,11} \mathrm{y}$ deberían formar parte de la prescripción con receta electrónica y estar integradas en la Historia Clínica electrónica.

Con este objetivo, se realizó un estudio observacional de no intervención, en el Hospital Ramón y Cajal con la herramienta informática GLIKAL, en el que se comparó las indicaciones que la herramienta sugería en base las guías clínicas introducidas, con los tratamientos propuestos por los médicos de urgencias (MU), los resultados demostraron que los MU no siguen las guías clínicas avaladas desde diferentes sociedades científicas ${ }^{12,13}$. Este estudio nos dejaba varias hipótesis que plantear: ¿es válida la aplicación GLIKAL y las guías clínicas que integra, en el tratamiento de pacientes con hiperglucemia?, ¿sería suficiente con la pauta de urgencias para la corrección de las cifras de glucemia en los días siguientes al ingreso?, ¿se modificaría el tratamiento propuesto desde urgencias en planta de hospitalización si las cifras de glucemia no son las adecuadas?. Con estas premisas previas, planteamos nuestro objetivo principal: demostrar superioridad de la aplicación informática GLIKAL (que utiliza algoritmos de las últimas guías clínicas de tratamiento de hiperglucemia en urgencias), en términos de reducción de cifras de glucemia tras una única intervención desde urgencias, en las primeras 48 horas tras el ingreso del paciente en planta de hospitalización. Para ello se propuso un ensayo clínico multicéntrico, de intervención en el que se pretendía demostrar la superioridad de GLIKAL frente a la práctica clínica habitual, comparando las medias de las glucemias de los 2 días posteriores al ingreso con el objetivo de que estas no superen los $180 \mathrm{mg} / \mathrm{dl}$.

\section{Materiales y Métodos}

\section{Diseño}

Ensayo clínico multicéntrico pragmático, en donde participaron 9 servicios de urgencias (SU) hospitalarios españoles, aleatorizado mediante un muestreo no probabilístico por conveniencia a una de las 2 ramas del estudio.

Se incluyeron pacientes desde octubre de 2013 a octubre de 2015, con los siguientes criterios de inclusión:

- Pacientes hombre o mujeres con hiperglucemia > $180 \mathrm{mg} / \mathrm{dl}$ (con o sin diagnóstico de DM).

- Edad comprendida entre 18 y 95 años de edad.

- Previsto ingreso de al menos 48 horas, con la posibilidad de realizar al menos 3 determinaciones de glucemia al día durante este tiempo.

- Firma del consentimiento informado.

Se excluyeron los pacientes con los siguientes criterios:

- Inestabilidad hemodinámica (tensión arterial media $<65 \mathrm{~mm} \mathrm{Hg}$ y/o tensión arterial sistólica $<90 \mathrm{~mm}$ $\mathrm{Hg})$.

- Necesidad de bomba de insulina.

- Cifras de glucemia $>399 \mathrm{mg} / \mathrm{dl}$.

- Pacientes que se vayan a someter a alguna cirugía los días posteriores.

- Pacientes quienes se hayan recogido menos de 3 determinaciones al día de glucemia en las 48 horas posteriores a su ingreso.

- Pacientes que según criterio del equipo médico se deban excluir.

- Pacientes que no firmaran el consentimiento informado.

Una vez valorado que el paciente cumplía con todos los criterios de inclusión y ninguno de los de exclusión, se le ofrecía participar en el estudio, que el paciente aceptaba mediante la firma del consentimiento informado.

\section{Intervención}

Los pacientes fueron aleatorizado mediante un muestreo no probabilístico por conveniencia a una de las 2 ramas del estudio:

- Práctica clínica habitual (PCH), sin intervención del investigador, en donde el tratamiento era pautado por otro médico del servicio ajeno al estudio.

- Rama experimental, en la que se utilizó la herramienta GLIKAL para prescribir el tratamiento. 
Todos los pacientes reclutados por los médicos pertenecientes al equipo investigador se incluían dentro de esta rama.

Los pacientes del grupo $\mathrm{PCH}$ se reclutaban en la sala de preingreso, o una vez ingresaban en las plantas de hospitalización, de forma que ya tuvieran pautado el tratamiento, y únicamente se recogían las variables de evaluación. Mientras que el grupo GLIKAL, se reclutaban desde su llegada a urgencias, se introducían los datos requeridos en el programa y se pautaba el tratamiento propuesto por el mismo, recogiéndose a posteriori las variables requeridas.

Variables de evaluación: La variable principal de evaluación, fue la media de las glucemias de los 2 días posteriores al ingreso, con el objetivo de que estas no superasen los $180 \mathrm{mg} / \mathrm{dl}$.

Se recogieron además datos demográficos (edad, sexo, peso) e información clínica (tipo de tratamiento antidiabético que recibía previo al ingreso, tratamiento actual con corticoides, si el paciente se mantenía en dieta absoluta, presencia o no de enfermedad renal y tipo, valor de creatinina actual, valor de glucemia a la llegada a urgencias), episodios de hipoglucemia en las primeras 48 horas tras el ingreso, y el número de días de hospitalización.

Recogida de datos: La recogida de datos se realizó en la propia aplicación GLIKAL, alojada en un servidor apropiado, los datos se guardaron con la seguridad propia del servidor, mediante encriptación de los mismos, garantizando el acceso únicamente al Investigador Principal (IP). Se proporcionó el acceso restringido a los investigadores colaboradores mediante clave de usuario y contraseña, cada investigador solo tuvo acceso a sus pacientes, mientras que el IP a todos los pacientes. En ningún caso se dejó guardada en la aplicación GLIKAL los datos de identificación de los pacientes.

Análisis estadístico: El tamaño muestral calculado inicialmente fue de 170 pacientes, 85 en cada rama de estudio para demostrar con un poder del $80 \%$ y un nivel alfa de 0.05 al menos un $15 \%$ de diferencia entre ambos grupos en cuanto al número de pacientes que obtenían niveles de glucemia por debajo de $180 \mathrm{mg} / \mathrm{dl}$ en los dos primeros días de ingreso hospitalario. Se hizo un análisis por protocolo.

El análisis de la variable principal se hizo mediante la prueba de Chi-cuadrado obteniendo además la RR. Las diferencias en los datos demográficos y clínicos basales y las variables secundarias, se analizaron mediante la prueba de Chi cuadrado para las variables categóricas y la $\mathrm{T}$ de student o prueba $\mathrm{U}$ de MannWhitney para las variables continuas según prueba de normalidad. Se realizaron análisis univariantes para determinar las variables que se relacionaban con la obtención de una glucemia por debajo de $180 \mathrm{mg} / \mathrm{dl}$ en las primeras 24 y 48 horas tras el inicio de tratamiento, para posteriormente incluir aquellas con una $p<0,1$ en una regresión logística multivariante, además de incluir también aquellas variables consideradas clínicamente relevantes. Todos los contrastes fueron bilaterales con un nivel de significancia de 0.05 .

Aspectos éticos: Este ensayo clínico ha sido aprobado por el Comité Ético de Investigación Clínica del Hospital Ramón y Cajal. Al tratarse de un ensayo clínico regulado por la Ley de Investigación Biomédica (Ley 14/2007) no fue necesaria su aprobación por la Agencia Española de Medicamentos y Productos Sanitarios.

\section{Resultados}

Se reclutaron un total de 200 pacientes, de los cuales se retiraron 17 del análisis por no tener alguna de las determinaciones de glucemia (2 del grupo Glikal y 15 del grupo PCH), tal y como lo establece uno de los criterios de exclusión, quedando finalmente la cohorte de análisis de datos conformado por 183 pacientes; $86(47 \%)$ recibieron ajuste del tratamiento siguiendo la aplicación GLIKAL y 97 (53\%) según PCH (Fig 1). El 54.6\% de los pacientes eran hombres, con una edad de 77 (45-94) años y un peso de 77.4 (13.97) kg. Los pacientes previo al ingreso se encontraban en tratamiento en primer lugar con antidiabéticos orales (ADO) 37.2\%, seguido de tratamiento con insulina $33.9 \%$, en tercer lugar tratamiento mixto $19.1 \%$ y por último, el $9.8 \%$ de los pacientes no se encontraban con tratamiento farmacológico para la hiperglucemia. Se encontró diferencia estadísticamente significativa en la distribución entre los dos grupos (GLIKAL vs PCH) en el peso $(p=0.05)$ y en la presencia de enfermedad renal $(p=0.017)$. La tabla 1 muestra la distribución de las variables demográficas y clínicas en los dos grupos del estudio.

Fig 1. Esquema del número de pacientes reclutados y que hayan finalizado el estudio.

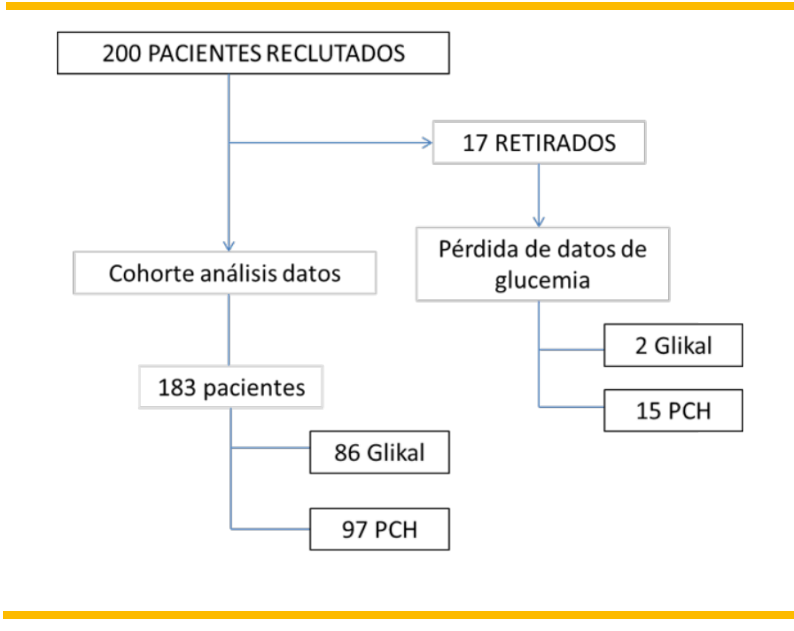

Variable principal

De los 86 pacientes del grupo GLIKAL, 54 (62.8\%) 
lograron mantener glucemia por debajo de $180 \mathrm{mg} / \mathrm{dl}$ en el primer día de ingreso hospitalario, frente a 32 pacientes $(33 \%)$ del grupo PCH $(\mathrm{p}<0.001)$. La diferencia de niveles de glucemia en el segundo día de ingreso, fue similar [58 (67.4\%) frente a $42(43.3 \%)$ ] $(\mathrm{p}=0.001)$. Ver figura 2 y tabla 2 .

Fig 2: Porcentaje de pacientes con glucemias medias diarias < $180 \mathrm{mg} / \mathrm{dl}$, en los 2 días siguientes al ingreso, en los dos grupos de estudio. GLIKAL: Rama de pacientes tratada con la aplicación GLIKAL. MED: Rama de pacientes tratada según $\mathrm{PCH}$.

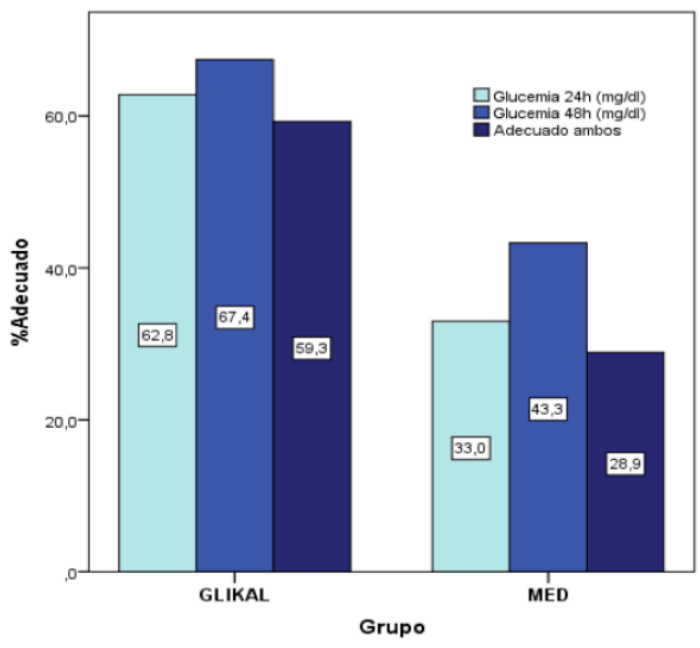

\section{Variables secundarias}

La media de glucemia en el primer día de ingreso en el grupo GLIKAL fue de 178.56 (59.53) frente a 212.93 (62.23) del grupo PCH $(\mathrm{p}<0.001)$ y en el segundo día de 173.86 (58.86) frente a 196.37 (66.60) $(p=0.017)$. No hubo diferencia en la frecuencia de hipoglucemia reportada en cada grupo $(\mathrm{p}=0.555)$. Aunque no era uno de los objetivos del estudio, se observó una reducción de estancia media en los pacientes tratados con GLIKAL. Los días de ingreso fueron de 7 (2-39) días para el grupo GLIKAL y de 10 (2-53) días para el grupo PCH $(\mathrm{p}<0.001)$ (Fig 3).

\section{Análisis univariante}

Las variables relacionadas con obtener valores de glucemia $<180 \mathrm{mg} / \mathrm{dl}$ en el primer y segundo día, fueron: el control de las cifras mediante el uso de la aplicación GLIKAL o PCH $(\mathrm{p}=0.001)$, y el tratamiento que recibía el pacientes previo a su llegada a urgencias ( $p=0.041$ y 0.010 respectivamente).

\section{Análisis multivariante}

Se realizó una regresión logística, para determinar las variables que de forma conjunta están relacionadas con tener o no valores de glucemia por debajo de 180 $\mathrm{mg} / \mathrm{dl}$, en el primer y segundo día de ingreso. En función del análisis univariante se seleccionaron las variables independientes $(\mathrm{p}<0.10)$ a incluir en el modelo. Finalmente, se incluyó el grupo al que ha sido aleatorizado el paciente, el tratamiento previo al ingreso a urgencias y se ajustó también al valor de la glucemia a la llegada a urgencias. En el análisis de las primeras 24 horas, queda únicamente el grupo al que se destinó al paciente (GLIKAL vs PCH), como variable con significación estadística. Mientras que en el análisis del segundo día, se relaciona tanto el grupo al que ha sido aleatorizado como el tratamiento que recibía el paciente antes de su ingreso a urgencias.

Fig 3: Días de ingreso en los dos grupos de estudio. GLIKAL: Rama de pacientes tratada con la aplicación GLIKAL. MED: Rama de pacientes tratada según PCH.

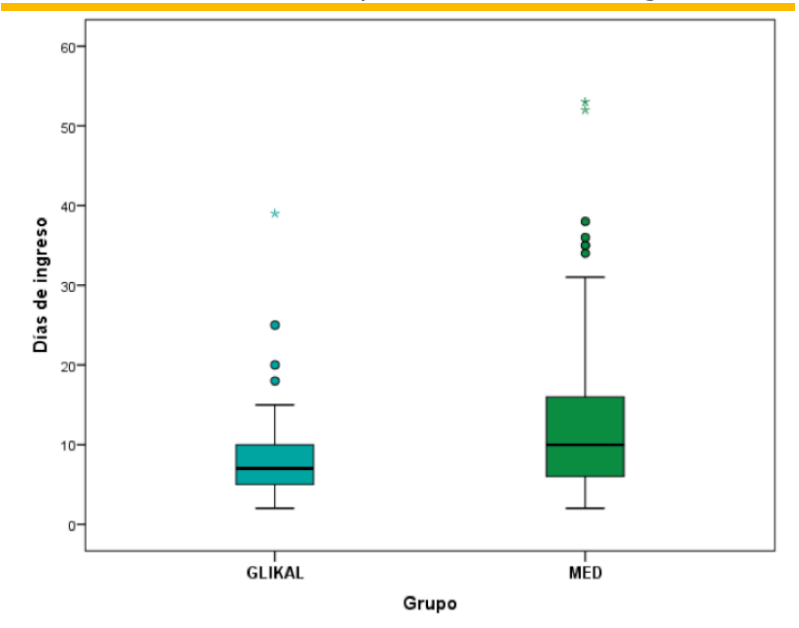

\section{Discusión}

El manejo de la hiperglucemia en los Servicios de Urgencias Hospitalaria (SUH) es un tema complejo y ampliamente estudiado $\left({ }^{10,11,14-20}\right)$. Se han establecido una gran cantidad de pautas, algoritmos, modelos, sin poder llegar a obtener una solución que sea altamente eficaz en su manejo, pudiendo incluso influir de forma importante la experiencia del médico a cargo del paciente. Todo esto hace que este manejo sea muy heterogéneo $\mathrm{y}$ exista gran variabilidad en los resultados.

Entre los factores que hace que el control de la hiperglucemia sea tan complicado está el riesgo de desarrollo de hipoglucemia, siendo éste el factor limitante para la optimización del control glucémico, ya que en muchas ocasiones lleva a un tratamiento subterapéutico. Tanto la hiper como la hipoglucemia conllevan a un aumento de la morbi-mortalidad en pacientes críticos, por lo que hace que un control glucémico eficaz y precoz sea primordial desde su llegada a los SUH.

Según nuestra búsqueda, aunque hay varios estudios de manejo de la glucemia en paciente diabéticos con aplicaciones informáticas ${ }^{9-11,21-23}$, este es el primer ensayo clínico realizado en urgencias, que 
valida una aplicación informática en la ayuda al tratamiento de la hiperglucemia. Los resultados obtenidos en este estudio indican, que la utilización de GLIKAL como apoyo a los MU en la decisión de tratamiento de paciente con hiperglucemia, es segura y eficiente, y que su uso debería estandarizarse para asegurar el cumplimiento de las guías clínicas, lo que redundaría en un mejor control de las cifras de glucemia en los pacientes ingresados, con los beneficios que el control de las cifras de glucemias por herramientas informáticas de ayuda al clínico, han demostrado en numerosas publicaciones previas ${ }^{14,21}$.

Una de las principales ventajas de la aplicación, es que fue utilizada por los profesionales sin ningún tipo de aprendizaje previo a la realización del estudio, lo que demuestra el manejo intuitivo de la herramienta, siendo ideal para la utilización en todo tipo de entornos y por todo tipo de profesionales (adjuntos, residentes, enfermería..etc). Ver sencillez de manejo en la introducción de datos en figura 4.

Fig 4: Pantalla ejemplo de la aplicación GLIKAL.

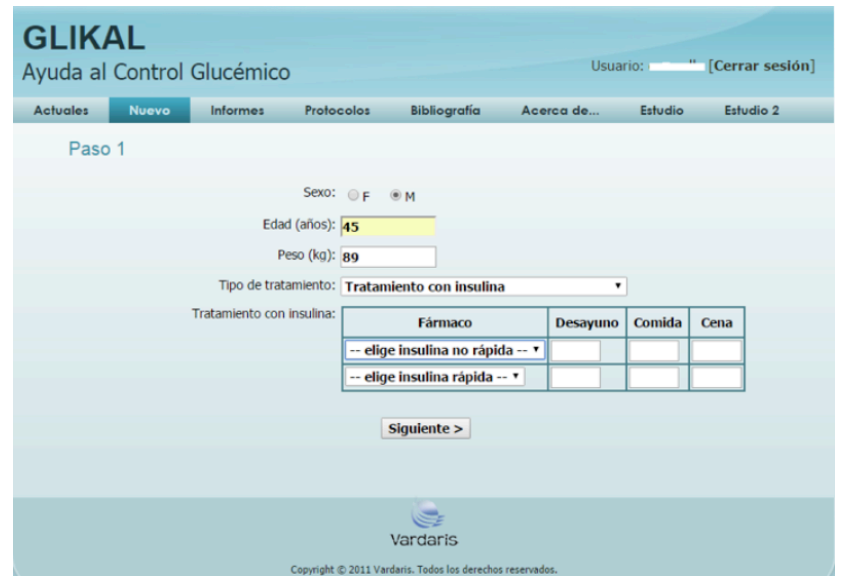

En el análisis vemos que hubo más pacientes que lograron el objetivo propuesto, que es mantener una media de glucemia por debajo de $180 \mathrm{mg} / \mathrm{dl}$ en el primer y segundo día de ingreso hospitalario, en el grupo GLIKAL que en el grupo $\mathrm{PCH}$, que además, en el caso del primer día, la frecuencia prácticamente se duplica favoreciendo el ajuste del tratamiento mediante el uso de esta aplicación (62.8\% frente a 33\%). Además, el Riesgo Relativo obtenido en ambos días indican que el uso de la aplicación GLIKAL reduce entre 1.7 y 1.9 veces el riesgo de tener glucemia $>180$ $\mathrm{mg} / \mathrm{dl}$. Lo anterior se puede ver reflejado en la media de glucemia de ambos días. Los pacientes tratados mediante la aplicación GLIKAL, presentaron una media de glucemia más baja que los pacientes tratados con $\mathrm{PCH}$ (ver figura 2).

La seguridad de la aplicación GLIKAL, se vio reflejada en que la frecuencia de hipoglucemias reportadas es similar a las del grupo $\mathrm{PCH}$. La hipoglucemia es considerada el acontecimiento adverso más frecuente de los protocolos de manejo de hiperglucemia, y saber que el uso de GLIKAL no aumenta su frecuencia aumenta la confianza de los clínicos en su uso.

Los pacientes tratados mediante la aplicación GLIKAL presentaron menor estancia hospitalaria que los pacientes del grupo PCH (ver figura 3). Este resultado es interesante dentro del Sistema Nacional de Salud, ya que ayudaría a reducir los gastos en concepto de ingreso hospitalario.

La glucemia a la llegada a urgencias no presentó en ninguno de los dos días significancia estadística. Esto se explica por el hecho que las glucemias iniciales fueron similares en los dos grupos y no influyó en la respuesta terapéutica.

Entre las limitaciones del ensayo clínico, se encuentra el tipo de aleatorización que se tuvo que elegir, con el objetivo de asegurar que la pauta según $\mathrm{PCH}$ no se viera influenciada por la participación del paciente en el ensayo clínico. Así mismo, las pérdidas que tuvimos en el seguimiento, se debió a que el estudio se llevó a cabo en el contexto de la $\mathrm{PCH}$, siendo mínima la intervención de los investigadores.

En conclusión, nuestros datos demuestran que GLIKAL es una aplicación segura y que ha demostrado ser superior a la práctica clínica habitual en los pacientes que ingresan desde el SU, permitiendo un mejor control de las cifras de glucemia en los días posteriores al ingreso, lo que acorta la estancia media de los pacientes ingresados, con un índice de hipoglucemias similar al tratamiento convencional.

\section{Agradecimiento}

Este trabajo ha contado con el apoyo de la Plataforma Española de Investigación Clínica y Ensayos Clínicos, SCReN (Spanish Clinical Research Network), financiada por el ISCIII-Subdirección General de Evaluación y Fomento de la Investigación, mediante el proyecto PT13/0002/0015, integrado en el Plan Estatal de I+D+I 2013-2016 y cofinanciado por y el Fondo Europeo de Desarrollo Regional (FEDER).

\section{Bibliografía}

[1] Soriguer F, Goday A, Bosch-Comas A, Bordiú E, Calle-Pascual A, Carmena R, et al. Prevalence of diabetes mellitus and impaired glucose regulation in Spain: the Di@bet.es Study. Diabetologia 2012;55:88-93.

[2] Ibarra A. Prevalencia y características clínicas de pacientes diabéticos ingresados en un hospital general. Arch Med Interna 2015;37:57-60.

[3] González P, Faure E, del Castillo A. Coste de la diabetes mellitus en España. Medicina Clínica 2006; 127:776-884. 
[4] Ballesta MJ, Carral F, Olveira G, Girón JA, Aguilar M. Economic costs associated with type II diabetes in Spanish patients. Eur J Health Econ 2006; 7:270-5.

[5] Hidalgo A, Oliva J, Rubio M, Zozaya N, Villoro $\mathrm{R}$, García S. "Estudios de coste de la diabetes tipo 2: una revisión de la literatura". Agencia de Evaluación de Tecnologías Sanitarias Instituto de Salud Carlos III - Ministerio de Economía y Competitividad 2015.

[6] Boord JB, Greevy RA, Braithwaite SS, Arnold PC, Selig PM, Brake $\mathrm{H}$, et al. Evaluation of Hospital Glycemic Control at US Academic Medical Centers. J Hosp Med 2009;4:35-44.

[7] Crespo C, Brosa M, Soria-Juan A, Lopez-Alba A, López-Martínez N, Soria B. Costes directos de la diabetes mellitus y de sus complicaciones en España (Estudio SECCAID: Spain estimated cost Ciberdem Cabimerin Diabetes). Av Diabetol 2013;29:182-9.

[8] Consejería de Sanidad. Estrategia de Atención a Pacientes con Enfermedades Crónicas en la Comunidad de Madrid. 2013:109.

[9] Katalenich B, Shi L, Liu S, Shao H, McDuffie R, Carpio G, et al. Evaluation of a Remote Monitoring System for Diabetes Control. Clin Ther 2015;37:1216-25.

[10] Aloi J, Bode BW, Ullal J, Chidester P, McFarland $\mathrm{RS}$, Bedingfield AE, et al. Comparison of an Electronic Glycemic Management System Versus Provider-Managed Subcutaneous Basal Bolus Insulin Therapy in the Hospital Setting. J Diabetes Sci and Technol. 2017;11:12-16.

[11] Sáenz A, Brito M, Morón I, Torralba A, GarcíaSanz E, Redondo J. Development and Validation of a Computer Application to Aid the Physician's Decision-Making Process at the Start of and during Treatment with Insulin in Type 2 Diabetes: A Randomized and Controlled Trial. J Diabetes Sci and Technol 2012;6:581-8.

[12] Carballo Cardona C, Gallego Rodríguez P, W Alba L, Zamorano Serrano M, Estévez Rueda MJ, Fernández-Pérez C. Adherencia de los Urgenciólogos al protocolo de Control Glucémico de la Herramienta GLIKALC. Journal of negative and no positive results 2016;1:1-7.

[13] Alvarez Rodriguez E, Agud fernández M, Caurel Sastre Z, Gallego Mínguez I, Carballo Cardona C,
Juan Arribas A, et al. Recomendaciones de manejo de la diabetes, de sus complicaciones metabólicas agudas y de la hiperglucemia relacionada con corticoides en los servicios de urgencias. Emergencias 2016;28:400-17.

[14] Boaz M, Landau Z, Wainstein MJ. Analysis of Institutional Blood Glucose Surveillance. J Diabetes Sci and Technol 2010;4:1514-5.

[15] Whitmore C. Blood glucose monitoring: an overview. Br J Nurs 2012;21:583-7.

[16] Strippoli GF, Di Paolo S, Cincione R, Di Palma AM, Teutonico A, Grandaliano G, et al. Clinical and therapeutic aspects of diabetic nephropathy. J Nephrol 2003;16:487-99.

[17] Clement S, Braithwaite SS, Magee MF, Ahmann A, Smith EP, Schafer RG, et al. Management of diabetes and hyperglycemia in hospitals. Diabetes Care 2004;27:553-97.

[18] Shimizu T, Nathan DM, Buse JB, Davidson MB, Ferrannini E, Holman RR, et al. Medical management of hyperglycemia in type 2 diabetes: a consensus algorithm for the initiation and adjustment of therapy: a consensus statement of the American Diabetes Association and the European Association for the Study of Diabetes. Nihon Rinsho 2012;70 Suppl 3:591-601.

[19] GRUPO SEMES DIABETES. Manejo del paciente diabético en urgencias. http://wwwsemesdiabeteses/protocolo_diabetes_2 pdf 2013.

[20] Carreño Hernández MC, Sabán Ruiz J, Fernández Ballesteros A, Bustamante Fermose A, García Polo I, Guillén Camargo V, et al. Manejo del paciente diabético hospitalizado. An Med Interna 2005;22:339-48.

[21] Ampudia-Blasco FJ, Benhamou PY, Charpentier G, Consoli A, Diamant M, Gallwitz B, et al. A Decision Support Tool for Appropriate GlucoseLowering Therapy in Patients with Type 2 Diabetes. Diabetes Technol Ther 2015;17:194202.

[22] Breault JL, Goodall CR, Fos PJ. Data mining a diabetic data warehouse. Artif Intell Med 2002;26:37-54.

[23] Cebul RD, Love TE, Jain AK, Hebert CJ. Electronic Health Records and Quality of Diabetes Care. N Engl J Med 2011:825-33. 
Tabla 1. Características demográficas y clínicas de los pacientes por grupo de estudio.

\begin{tabular}{|c|c|c|c|}
\hline VARIABLES & GLIKAL & PCH & $\mathbf{p}$ \\
\hline $\mathrm{n}(\%)$ & $86(47 \%)$ & $97(53 \%)$ & - \\
\hline \multicolumn{4}{|l|}{$\mathrm{SEXO}, \mathrm{n}(\%)$} \\
\hline HOMBRE & $46(53.5 \%)$ & $54(55.7 \%)$ & \multirow{2}{*}{0.767} \\
\hline MUJER & $40(46.5 \%)$ & $43(44.3 \%)$ & \\
\hline EDAD; mediana (rango) & $77(45-91)$ & $76.5(46-94)$ & 0.797 \\
\hline $\mathrm{PESO} ; \bar{x}(\mathrm{DE})$ & $79.55(13.42)$ & $75.49(14.23)$ & 0.050 \\
\hline \multicolumn{4}{|l|}{ TTO CORTICOIDES } \\
\hline $\mathrm{SI} / \mathrm{NO}$ & $30(34.9 \%) / 56(65.1 \%)$ & $26(26.8 \%) / 71(73.2 \%)$ & 0.237 \\
\hline \multicolumn{4}{|l|}{ DIETA ABSOLUTA } \\
\hline $\mathrm{SI} / \mathrm{NO}$ & $18(20.9 \%) / 68(79.1 \%)$ & $11(11.3 \%) / 86(88.7 \%)$ & 0.076 \\
\hline \multicolumn{4}{|l|}{ TRATAMIENTO PREVIO, n (\%) } \\
\hline NINGUNO & $9(10.5 \%)$ & $9(10.5 \%)$ & \multirow{4}{*}{0.429} \\
\hline $\mathrm{ADO}^{*}$ & $34(39.5 \%)$ & $34(35.1 \%)$ & \\
\hline INSULINA & $24(27.9 \%)$ & $38(39.2 \%)$ & \\
\hline MIXTO & $19(22.1 \%)$ & $16(16.5 \%)$ & \\
\hline \multicolumn{4}{|l|}{ ENFERMEDAD RENAL, n (\%) } \\
\hline NO & $67(77.9 \%)$ & $59(60.8 \%)$ & \multirow{3}{*}{0.017} \\
\hline ENF RENAL MODERADA & $19(22.1 \%)$ & $34(35.1 \%)$ & \\
\hline ENF RENAL SEVERA & $0(0 \%)$ & $4(4.1 \%)$ & \\
\hline GLUCEMIA ENTRADA, $\bar{x}$ (DE) & $260.91(49.78)$ & $261.86(56.28)$ & 0.905 \\
\hline CREATININA; $\overline{\mathbf{x}}$ (DE) & $1.30(0.62)$ & $1.38(0.79)$ & 0.455 \\
\hline
\end{tabular}

*ADO: Antidiabético oral

Tabla 2. Variables de evaluación por grupo de estudio

\begin{tabular}{|c|c|c|c|c|c|}
\hline VARIABLES & GLIKAL & $\mathrm{PCH}$ & $\begin{array}{l}\text { DIF } \\
\text { MEDIA }\end{array}$ & IC $95 \%$ & $\mathrm{P}$ \\
\hline GLUCEMIA PRIMER DIA; $\bar{x}(\mathrm{DE})$ & $\begin{array}{l}178.56 \\
(59.53)\end{array}$ & $212.93(68.23)$ & 34.37 & $15.58-53.16$ & $<0.001$ \\
\hline $\begin{array}{l}\text { GLUCEMIA SEGUNDO DÍA; } \bar{x} \\
\text { (DE) }\end{array}$ & $\begin{array}{l}173.86 \\
(58.86)\end{array}$ & $196.37(66.60)$ & 22.51 & $4.07-40.95$ & 0.017 \\
\hline \multicolumn{6}{|l|}{ HIPOGLUCEMIA, n (\%) } \\
\hline NO & $82(95.3 \%)$ & $93(95.9 \%)$ & & & \\
\hline MODERADA & $3(3.5 \%)$ & $4(4.1 \%)$ & & & 0.555 \\
\hline SEVERA & $1(1.2 \%)$ & $0(0 \%)$ & & & \\
\hline $\begin{array}{l}\text { DÍAS DE INGRESO, mediana } \\
\text { (rango) }\end{array}$ & $7(2-39)$ & $10(2-53)$ & 5.235 & $2.816-7.654$ & $<0.001$ \\
\hline
\end{tabular}

